\title{
Cycloidal Domains in the Magnetization Reversal Process of $\mathrm{Ni}_{80} \mathrm{Fe}_{20} / \mathrm{Nd}_{16} \mathrm{Co}_{84} / \mathrm{Gd}_{12} \mathrm{Co}_{88}$ Trilayers
}

\author{
C. Quiros, ${ }^{1,2}$ A. Hierro-Rodriguez,${ }^{3, \dagger}$ A. Sorrentino, ${ }^{3}$ R. Valcarcel,${ }^{3}$ L. M. Alvarez-Prado,,${ }^{1,2}$ \\ J. I. Martín, ${ }^{1,2}$ J. M. Alameda, ${ }^{1,2}$ E. Pereiro, ${ }^{3}$ M. Vélez, ${ }^{1,2,{ }^{*}}$ and S. Ferrer ${ }^{3}$ \\ ${ }^{1}$ Departamento de Física, Universidad de Oviedo, 33007 Oviedo, Spain \\ ${ }^{2}$ CINN (CSIC - Universidad de Oviedo), 33940 El Entrego, Spain \\ ${ }^{3}$ ALBA Synchrotron, 08290 Cerdanyola del Vallès, Spain
}

(Received 6 February 2018; revised manuscript received 18 April 2018; published 12 July 2018)

\begin{abstract}
The magnetization reversal of each individual layer in magnetic trilayers (permalloy/NdCo/GdCo) is investigated in detail with x-ray microscopy and micromagnetic calculations. Two sequential inversion mechanisms are identified. First, magnetic vortex-antivortex pairs move along the field direction while inverting the magnetization of magnetic stripes until they are pinned by defects. The vortex-antivortex displacements are reversible within a field interval which allows their controlled motion. Second, as the reversed magnetic field increases, cycloidal domains appear in the permalloy layer as a consequence of the dissociation of vortex-antivortex pairs due to pinning. The field range where magnetic vortices and antivortices are effectively guided by the stripe pattern is of the order of tens of $\mathrm{mT}$ for the NiFe layer, as estimated from the stability of cycloid domains in the sample.
\end{abstract}

DOI: 10.1103/PhysRevApplied.10.014008

\section{INTRODUCTION}

Three-dimensional magnetic textures nucleate in magnetic materials as a result of the competition of anisotropy, exchange, and magnetostatic interactions. Skyrmions and helical domains occur in bulk materials with chiral exchange interactions [1,2] and also in films and nanostructures where their configuration can be tailored by confinement effects [3-5]. Noncollinear textures can also be stabilized by dipolar interactions in weak perpendicular magnetic anisotropy (WPMA) materials (e.g., dipolar skyrmions in $\mathrm{Gd} / \mathrm{Fe}$ [6] and dipolar merons in $\mathrm{NdCo}$ layers [7]) or by precessional dynamics [8]. Multilayered systems, combining materials with different exchange and anisotropy properties have been used to artificially imprint noncollinear magnetic textures in soft magnetic layers (e.g., artificial skyrmions [9], meron pairs [10], vortex-antivortex pairs [11]) and, also, to stabilize nontrivial spin configurations across the thickness such as topologically protected twisted magnetic helices in $\mathrm{Dy} / \mathrm{Fe}$ multilayers [12].

In WPMA layers [13], the equilibrium domain pattern consists of parallel up and down stripes separated by domain walls that change from Bloch (at the central plane)

\footnotetext{
*mvelez@uniovi.es

${ }^{\dagger}$ Present address: University of Glasgow, School of Physics and Astronomy, Glasgow G12 8QQ, United Kingdom.
}

to Néel (top and bottom surfaces) due to stray-field minimization. During in-plane magnetization reversal, Bloch

points (skyrmionic number $Q=1)$, merons $(Q=1 / 2)$ and magnetic vortex-antivortex pairs $(Q=1)$ nucleate at different sample depths and propagate guided by stripe direction $[7,11,14]$. In multilayers of significant thickness, strayfield circulation breaks the symmetry between top and bottom sample surfaces, providing a topological separation of magnetic textures in each layer [6,11]. In particular, magnetic vortex-antivortex nucleation is determined by topological characteristics (polarity, chirality, and bifurcation geometry) $[11,14]$, resulting in a robust mechanism to control the propagation of vortices and antivortices at top and bottom sample surfaces. Applications of this effect into memory devices based on the concept of magnetic vortex racetracks [15-17] would require a broad enough field range in which vortex motion can be reversibly controlled by an applied magnetic field and is effectively guided by the stripe-domain pattern. These issues require a detailed characterization of the field-dependent domain configuration of WPMA multilayers when vortex-antivortex pairs are present in the system.

A characteristic feature of such noncollinear spin systems is the presence of helical domains, stabilized by chiral magnetic interactions $[1,2,18]$. For thin films or in bulk materials close to sample surfaces, spin spirals can be modified by confinement effects and dipolar interactions that favor in-plane magnetization orientation [4,5]. Then, the 
most stable structures are formed by the combination of helical and cycloidal domains [Figs. 1(a) and 1(b)], as recently proposed and observed in chiral magnets $[4,5,19$ 22]. In a simple helical domain structure, periodic along $y$, the magnetization rotates $2 \pi \mathrm{rad}$ in the $M_{x}-M_{z}$ plane as sketched in Fig. 1(a). Close to an $x-y$ sample surface, magnetostatic effects confine the $2 \pi$ magnetization rotation to the $M_{x}-M_{y}$ plane, resulting in the cycloidal domain structure, also periodic along $y$, sketched in Fig. 1(b). In contrast, weak stripe-domain patterns, in the absence of chiral interactions, favor $\pi$ rotations of the magnetization around the average $M_{x}$ component, either in the $M_{x}-M_{z}$ plane (inside the film) or in the $M_{x}-M_{y}$ plane (at the top and bottom film surfaces). In the first case, this results in Bloch walls [Fig. 1(c)] while in the second case it results in closure domains at the top and bottom surfaces [Fig. 1(d)].

In this work, we report on the observation, for the first time, of cycloidal domains in soft permalloy layers using element-resolved magnetic transmission x-ray microscopy (MTXM). These domains are linked to the guided propagation of magnetic vortices and antivortices within the stripe-domain pattern of $\mathrm{NiFe} / \mathrm{NdCo} / \mathrm{GdCo}$ trilayers. The starting point is the remanent stripe-domain configuration illustrated in Fig. 2(a): the set of parallel up and down $\left(+M_{z}\right.$ and $\left.-M_{z}\right)$ domains in the central WPMA NdCo layer creates a closure domain pattern in both the top permalloy layer and the bottom GdCo layer. The magnetization in the permalloy layer oscillates mainly in the $\left(M_{x}, M_{y}\right)$ plane around the average $+M_{x}$ component (white arrow) to screen the stray field from the central NdCo layer. Upon the application of a reversed $-H_{x}$ field, cycloidal domains (sketched in the $M_{y}-M_{x}$ plane as $\rightarrow \uparrow \leftarrow \downarrow \rightarrow \uparrow$ ) are observed in the permalloy layer as an intermediate state between $+M_{x}\left(\rightarrow \uparrow \leftarrow \uparrow \rightarrow \uparrow\right.$ with only $+M_{x} \uparrow$ orientation) and $-M_{x}\left(\rightarrow \downarrow \leftarrow \downarrow \rightarrow\right.$ with only $\left.-M_{x} \downarrow\right)$ closure domains. Cycloidal domains in the permalloy layer are stable in a field range of several tens of $\mathrm{mT}$, which gives a measure of useful field range for controlled propagation of magnetic vortices and antivortices by the stripe-domain pattern.

\section{EXPERIMENT}

Magnetic $\mathrm{Ni}_{80} \mathrm{Fe}_{20} / \mathrm{Nd}_{16} \mathrm{Co}_{84} / \mathrm{Gd}_{12} \mathrm{Co}_{88}$ trilayers are grown on 50 -nm-thick $\mathrm{Si}_{3} \mathrm{~N}_{4}$ membranes by dc magnetron sputtering as reported previously [14] and characterized by vibrating sample magnetometry (VSM) at room temperature. Parameters for each material in the trilayer are obtained from the magnetic characterization of single test layers [14]: Permalloy $\mathrm{Ni}_{80} \mathrm{Fe}_{20}$ has $M_{S}=8.5 \times 10^{5}$ $\mathrm{A} / \mathrm{m}$, in-plane uniaxial magnetic anisotropy $K_{u}=850$ $\mathrm{J} / \mathrm{m}^{3}$, and a weak PMA of the order $K_{N} \approx 10^{4} \mathrm{~J} / \mathrm{m}^{3}$, induced by strains during growth but too low to induce out-of-plane domains in the samples investigated [10]; $\mathrm{Nd}_{16} \mathrm{Co}_{84}$ is an amorphous ferromagnetic alloy [23] with
$M_{S}=7 \times 10^{5} \mathrm{~A} / \mathrm{m}$ and PMA $K_{N} \approx 10^{5} \mathrm{~J} / \mathrm{m}^{3} ; \mathrm{Gd}_{12} \mathrm{Co}_{88}$ is a ferrimagnetic alloy with $M_{S}=6 \times 10^{5} \mathrm{~A} / \mathrm{m}$ and in-plane $K_{u}=4.6 \times 10^{3} \mathrm{~J} / \mathrm{m}^{3}$ [24]. Two samples are used in this study with different layer thicknesses to check for reproducibility of the observed magnetic textures: F40N60G40 (40 nm Ni ${ }_{80} \mathrm{Fe}_{20} / 60 \mathrm{~nm} \mathrm{Nd}{ }_{16} \mathrm{Co}_{84} / 40 \mathrm{~nm} \mathrm{Gd}{ }_{12} \mathrm{Co}_{88}$ ) and

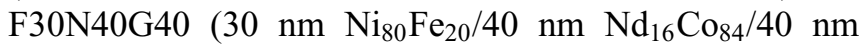
$\mathrm{Gd}_{12} \mathrm{Co}_{88}$ ). Figures 2(b) and 2(c) show the VSM hysteresis loops of the trilayers with the applied field parallel to the sample plane. In both cases, the hysteresis loops present a transcritical shape, characterized by an almost linear decrease of in-plane magnetization from saturation (at around $200 \mathrm{mT}$ ) to a reduced remanence value (about $0.4 M_{S}$ ), which is typical of WPMA samples with parallel stripe-domain patterns (i.e., with a significant out-of-plane magnetization oscillation). The coercivity $\mu_{0} H_{C} \approx 10 \mathrm{mT}$ is very similar in both samples [see insets in Figs. 2(b) and 2(c)], but the reversal process is broader for F30N40G40 $\left(\mu_{0} \Delta H=20 \mathrm{mT}\right)$ than for F40N60G40 $\left(\mu_{0} \Delta H=6 \mathrm{mT}\right)$, indicating a stronger effect of the $\mathrm{NdCo}$ layer in the thinner NiFe layer of F30N40G40.

Element-specific magnetic contrast images $[7,14]$ are acquired at the transmission microscope of the Mistral Beamline at the Alba synchrotron $[25,26]$ by tuning circularly polarized $\mathrm{x}$ rays to the appropriate atomic absorption energy of that element. MTXM images of the magnetization of the upper permalloy layer are acquired at the $L_{3}$ Fe absorption energy and of the bottom $\mathrm{Gd}_{12} \mathrm{Co}_{88}$ at the $M_{5} \mathrm{Gd}$ absorption energy [14]. At room temperature, due to the ferrimagnetic nature of this alloy for this relative Co to rare-earth concentration, the Gd magnetic moment is antiparallel to the sample magnetization, which has to be taken into account in the interpretation of magnetic contrast images [24]. The x-ray angle of incidence is set at $\theta=30^{\circ}$ from the film normal, in order to have sensitivity to $M_{x}$ and $M_{z}$ magnetization components while keeping good image qualities not hindered by the decreased transmitted x-ray intensities at large angles [7]. The sample is first saturated with an in-plane field $\mu_{0} H_{x}=225 \mathrm{mT}$ and imaged at remanence to establish the initial $+M_{x}$ magnetization state. Then, negative $H_{x}$ pulses of $20 \mu$ s duration and variable amplitude are applied in situ to monitor the magnetization reversal. The samples are imaged at remanence after each field pulse until in-plane magnetization is fully reversed.

Micromagnetic simulations of magnetization reversal are performed with the finite-difference code MUMAX ${ }^{3}$ [27] in order to compare with experimental MTXM images. The trilayers are discretized into cells of dimensions of $5 \times 5 \times 3 \mathrm{~nm}^{3}$ for a total area of $3.84 \times 3.84$ $\mu \mathrm{m}^{2}$, using material parameters for each sublayer obtained from the magnetic characterization [13]. MUVIEW code is used for visualization [28]. Then, the contribution to the dichroic absorption factor of each cell along the x-ray beam path is evaluated for the calculated micromagnetic 


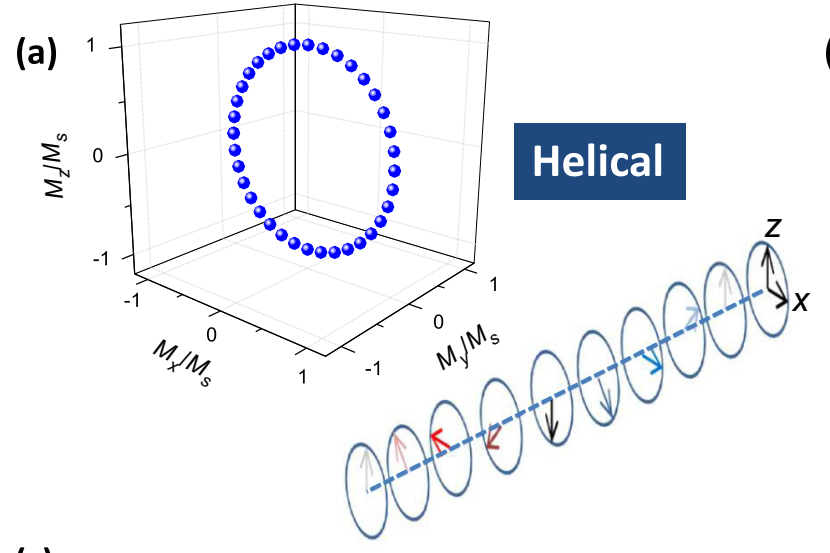

(c)

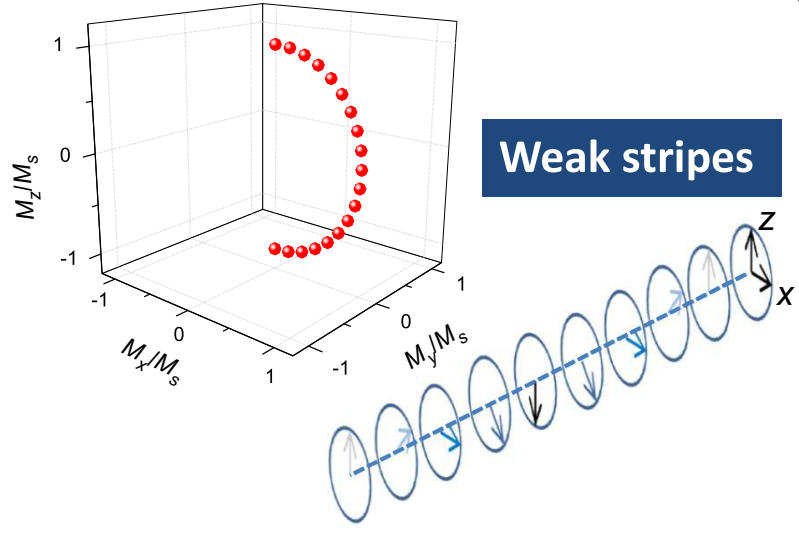

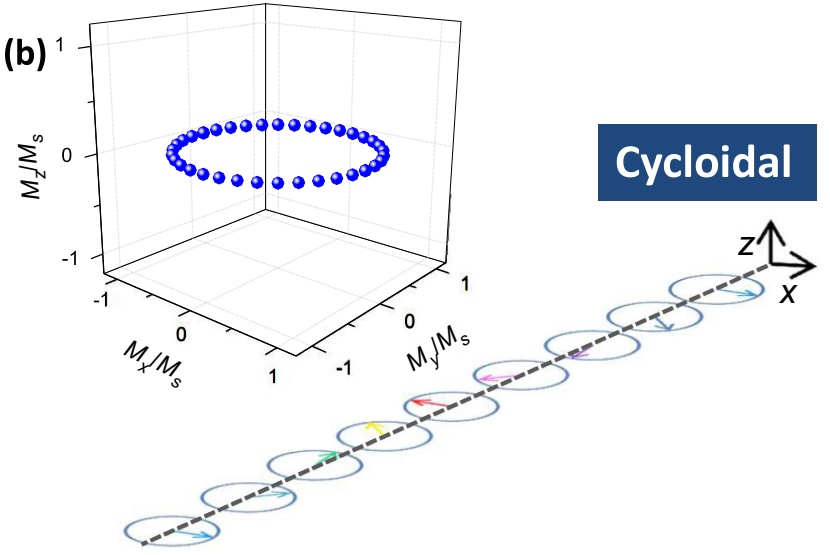

(d)

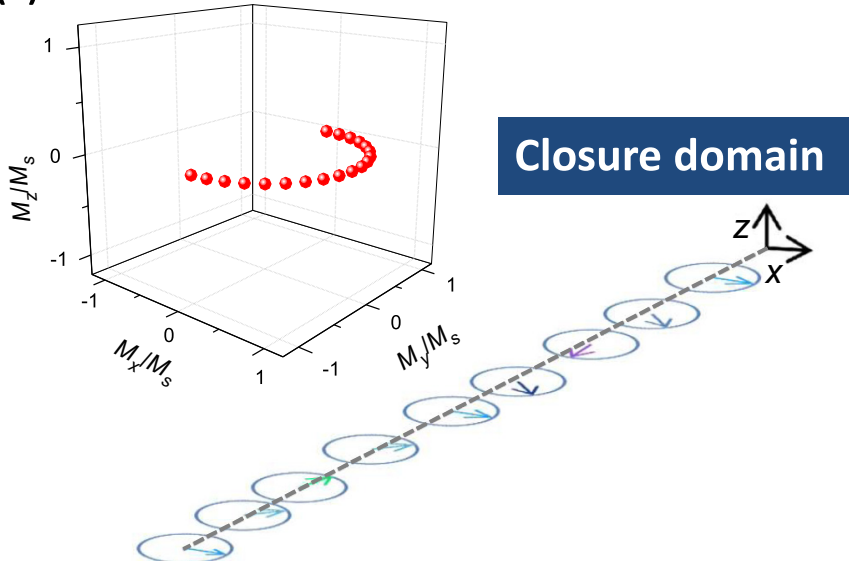

FIG. 1. Sketch of spin rotation within a domain period in: (a) $2 \pi$ helical domain in the $M_{x}-M_{z}$ plane, (b) $2 \pi$ cycloidal domain in the $M_{x}-M_{y}$ plane, (c) Bloch walls at the center of a weak stripe pattern ( $\pi$ rotation in the $M_{x}-M_{z}$ plane from a $+M_{z}$ domain to a $-M_{z}$ domain through a Bloch wall with $+M_{x}$ at its center), and (d) closure domains at the surfaces of a weak stripe-domain pattern ( $\pi$ rotation in the $M_{x}-M_{y}$ plane from a $+M_{y}$ domain to a $-M_{y}$ domain through a Néel wall with $+M_{x}$ at its center). In all cases, the domain structures are periodic along $y$.
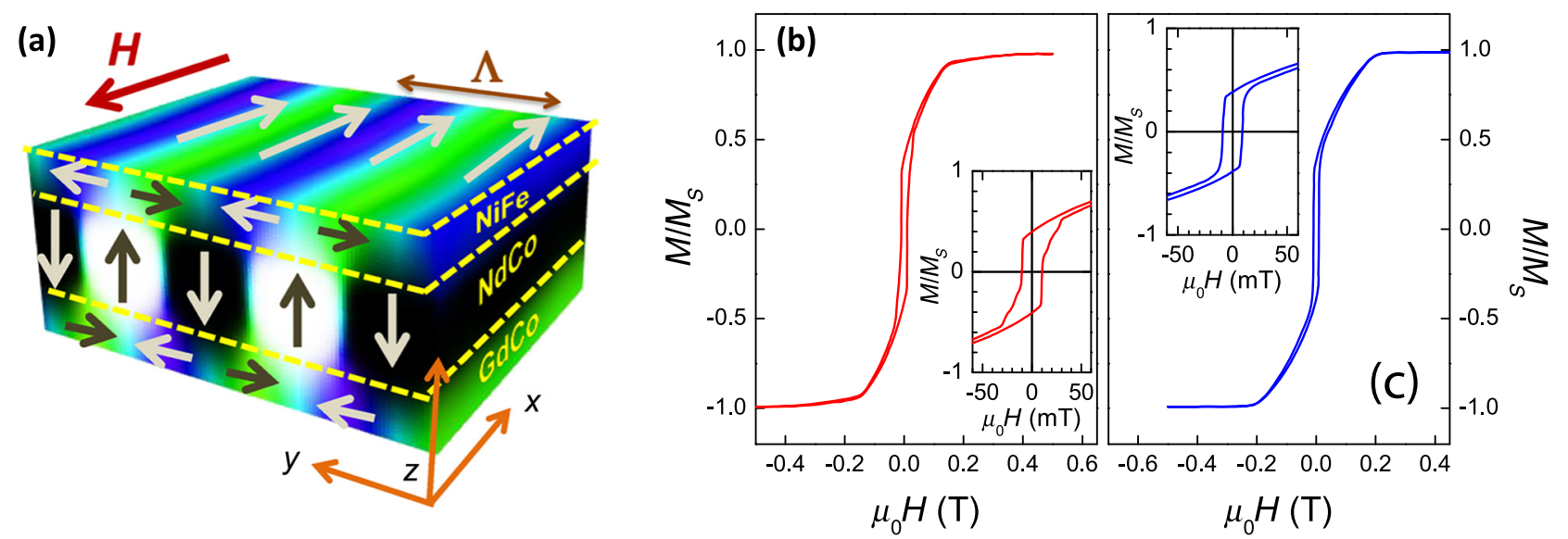

FIG. 2. (a) Sketch of stripe-domains in a $\mathrm{NiFe} / \mathrm{NdCo} / \mathrm{GdCo}$ trilayer at remanence after saturating with $+H_{x}$. The stripe-domain period $\Lambda$ is indicated by the double arrow. (b) VSM in-plane hysteresis loop of the F30N40G40 trilayer. (c) VSM in-plane hysteresis loop of the F40N60G40 trilayer. Insets show the low-field enlargements of the hysteresis loops. 
configurations in order to simulate $\mathrm{x}$-ray image contrasts [11].

\section{RESULTS AND DISCUSSION}

\section{A. MTXM hysteresis loops}

Figure 3 shows several MTXM frames of a $12 \times 12 \mu \mathrm{m}^{2}$ area of sample F40N60G40 imaged at the $\mathrm{L}_{3}$ Fe edge (left panels) and $\mathrm{M}_{5} \mathrm{Gd}$ edge (right panels) along a sequence of reversed field pulses of increasing amplitude. Figure 3(a) shows the onset of magnetization reversal at both $\mathrm{NiFe}$ and GdCo layers: most of the image is covered by a pattern of bright and dark parallel stripes oriented along the $x$ direction of period $\Lambda=175 \mathrm{~nm}$. In this sample x-ray geometry [7], image contrast depends on $M_{x}$ and $M_{z}$ and is not sensitive to $M_{y}$ : in the permalloy layer [see inset in the left panel of Fig. 3(a)], white stripes correspond to $\left(+M_{x},+M_{z}\right)$ domains and dark gray stripes to $\left(+M_{x},-M_{z}\right)$ domains as indicated by arrows; in the GdCo layer, due to the ferrimagnetic character of the alloy, dark gray bands correspond to $\left(+M_{x},+M_{z}\right)$ and light gray bands to $\left(+M_{x},-M_{z}\right)$ [14]. This corresponds to a remanent domain pattern similar to the sketch in Fig. 2(a) with average in-plane magnetization orientation along $+M_{x}$. In Fig. 3(a), we can also observe some additional contrast levels indicating the presence of initial reversed domains (after the application of a field pulse of $-22.5 \mathrm{mT}$ ) with a characteristic elongated shape: they typically consist of a pair of reversed consecutive up and down stripes [see D1 in Fig. 3(a)] starting at a bifurcation of the stripe pattern. These domains correspond to the nucleation of a meron texture at the bifurcation core (with $Q=1 / 2$ ) and the propagation of Bloch points and vortex-antivortex pairs (with $Q=1$ ) along the stripe direction [7,11]. At $\mu_{0} H_{x}=-25 \mathrm{mT}$ [Fig. 3(b)], magnetization reversal has proceeded with the nucleation of new reversed domains and the propagation of existing ones: at the $\mathrm{NiFe}$ layer, reversed domains retain their elongated shape extending only over one or two stripe-domain periods (average domain width is $1.2 \Lambda$ ), however, at the GdCo layer, reversed domains have grown laterally (e.g., see D2) with average domain width $4.5 \Lambda$. Finally, after a $\mu_{0} H_{x}=-32.5 \mathrm{mT}$ pulse [Fig. 3(c)], reversal is completed in the GdCo layer whereas the magnetic configuration of the NiFe layer is highly inhomogenous. Four contrast levels can be clearly observed in the NiFe panel of Fig. 3(c): white, light gray, dark gray, and black, corresponding to the four possible domain combinations: $\left(+M_{x},+M_{z}\right)$, $\left(-M_{x},+M_{z}\right),\left(+M_{x},-M_{z}\right)$, and $\left(-M_{x},-M_{z}\right)$, respectively (see arrows in the sketch) [14].

In a stripe pattern with uniform $M_{x}$ sign (as the one sketched in Fig. 2), magnetic dichroic contrast oscillates in the up and down domains around a mean value given by the average $M_{x}$ component [7]. Thus, we can follow in-plane magnetization reversal in more detail by plotting the average image contrast as a function of reversed field amplitude [Fig. 3(d)]. For both NiFe and GdCo layers, magnetization reversal starts at a similar field around $-22 \mathrm{mT}$, but reversal is much easier in the GdCo layer (it is completed in $\mu_{0} \Delta H \approx 5 \mathrm{mT}$ ) than in the NiFe layer $\left(\mu_{0} \Delta H \approx 25 \mathrm{mT}\right)$, indicating that expansion of reversed domains is more difficult in the NiFe layer.

Figure 4 shows a similar sequence of MTXM frames of a $12 \times 12 \mu \mathrm{m}^{2}$ area of sample F30N40G40 imaged at the $\mathrm{L}_{3}$ Fe edge (left panels) and $\mathrm{M}_{5} \mathrm{Gd}$ edge (right panels) along the magnetization reversal process of both $\mathrm{NiFe}$ and $\mathrm{GdCo}$ layers. Once again, magnetization reversal is much easier in the GdCo layer than in the NiFe layer. Bottom GdCo reverses in a narrow field interval $\left(\mu_{0} \Delta H \approx 5 \mathrm{mT}\right)$ by the lateral expansion of wide domains that extend over several stripe periods. Reversal at the top NiFe layer spans over $\mu_{0} \Delta H \approx 46 \mathrm{mT}$, with a $12 \mathrm{mT}$ plateau close to the coercivity [see Fig. 4(d)]. At the initial stages of magnetization reversal in this NiFe layer $\left[\mu_{0} H_{x} \approx-25 \mathrm{mT}\right.$, Fig. 4(a)], linear reversed domains are observed that expand along the stripe direction, as in the previous sample. Domain configuration at the plateau $\left(-40 \mathrm{mT}<\mu_{0} H_{x}<-28 \mathrm{mT}\right)$ is characterized by the intermixing of stripes with opposite $M_{x}$ orientation [Fig. 4(b)]. Finally, $-M_{x}$ domains extending over several stripe-domain periods appear in the image for $\mu_{0} H_{x}<-40 \mathrm{mT}$ [Fig. 4(c)]. NiFe layer reversal is completed at $\mu_{0} H_{x} \approx-60 \mathrm{mT}$.

Thus, in both samples the field interval for magnetization reversal in the permalloy layer is significantly broader than in the GdCo layer (by a factor of 5-10), which might seem surprising in view of the softer magnetic behavior of simple permalloy layers. It suggests a crucial role of the stripe pattern imprinted by the $\mathrm{NdCo}$ central layer in the propagation of reversed domains in the top and bottom layers that requires a careful analysis of the MTXM images.

\section{B. Reversible domain expansion at the onset of magnetization reversal}

Figure 5 shows a detail of domain propagation at the onset of magnetization reversal in the bottom GdCo layer of F30N40G40, driven by a sequence of positive and negative field pulses. At the initial state [Fig. 5(a)], we can see the boundary between a $-M_{x}$ domain (lower part of the image) and a $+M_{x}$ domain (upper part of the image) that runs along a dark gray $\left(-M_{x},+M_{z}\right)$ and black $\left(+M_{x},+M_{z}\right)$ stripe [see dotted line in Fig. 5(a)]. It has been recently shown [11] that, due to geometrical restrictions by strayfield circulation and the periodicity of the stripe pattern, the magnetic texture that separates the dark gray $\left(-M_{x},+M_{z}\right)$ and the black $\left(+M_{x},+M_{z}\right)$ stripe portions at the bottom sample surface consists of a magnetic vortex with $+M_{z}$ polarity as inferred from micromagnetic simulations [see inset of Fig. 5(a)]. 
(a)

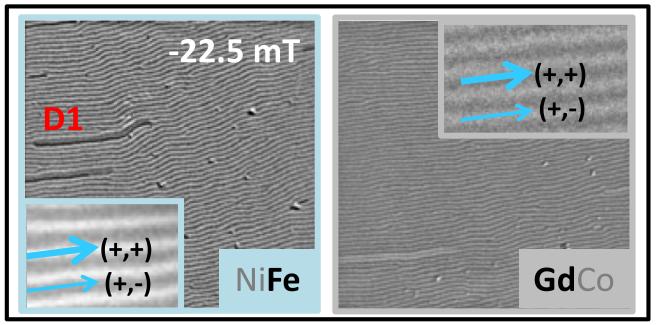

(b)

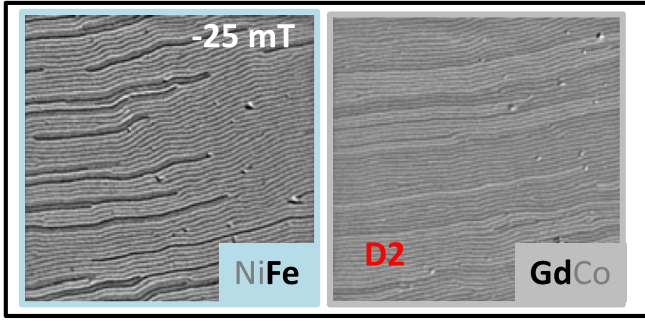

(c)

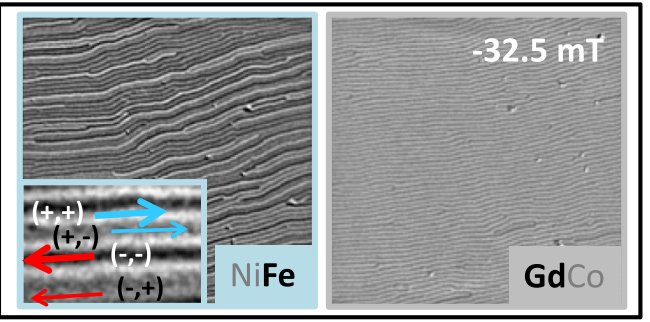

(d)

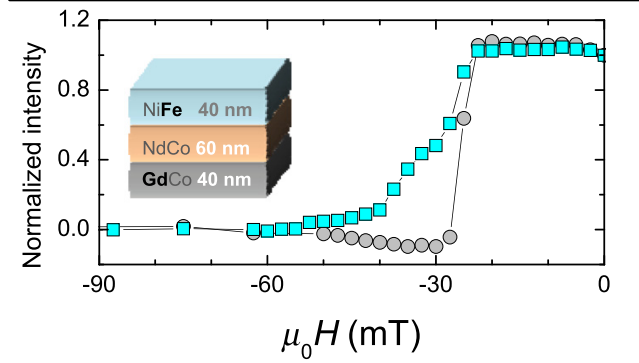

FIG. 3. (a)-(c) $12 \times 12 \mu \mathrm{m}^{2}$ MTXM frames of the magnetization configuration of F40N60G40 along in-plane magnetization reversal measured at the $\mathrm{L}_{3} \mathrm{Fe}$ edge (left panels) and the $\mathrm{M}_{5}$ Gd edge (right panels). Pulsed field amplitude is indicated in each image. Insets show sketches of magnetization orientation within the stripe pattern. (d) Field dependence of the normalized intensity of the Fe image (squares) and the Gd image (circles) obtained from experimental MTXM images that allow us to reconstruct element-selective hysteresis loops. Image intensities are normalized to +1 (at remanence) and to 0 (at saturation).

In the experiment depicted in Fig. 5, we observe the propagation of this magnetic vortex along the $+M_{z}$ stripe [dashed vertical lines in Fig. 5(a)], using as a reference the position of $Y_{1}$, the black bifurcation at the left of each panel [vertical solid line in Fig. 5(a)]. Also, in Fig. 5(a), the separation between bifurcations $Y_{1}$ and $Y_{2}$ is $4 \mu \mathrm{m}$, which we denote as $X_{\max }$. Figure 5(b) summarizes the results after applying successive field pulses. First, a set of equal amplitude $7.5 \mathrm{mT}$ pulses drove the vortex slowly to the right (increasing $X$ in the upper panel), until it stopped at the intermediate position $X \approx 0.3 X_{\max }$. Increasing pulse amplitudes up to $12 \mathrm{mT}$, depinned the vortex from this intermediate position and pushed it up to bifurcation $Y_{2}$ (the endpoint of this black stripe). Then, a set of negative field pulses of increasing amplitude is applied to the sample. No changes in the image are detected until (a)

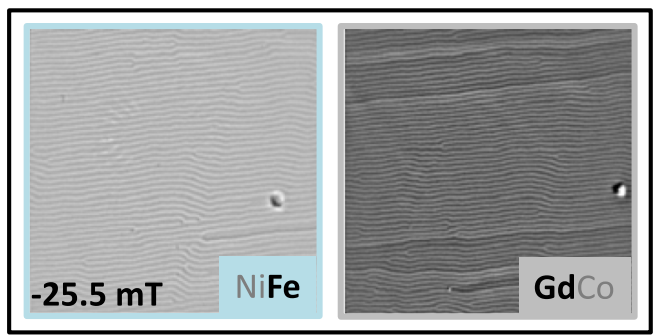

(b)

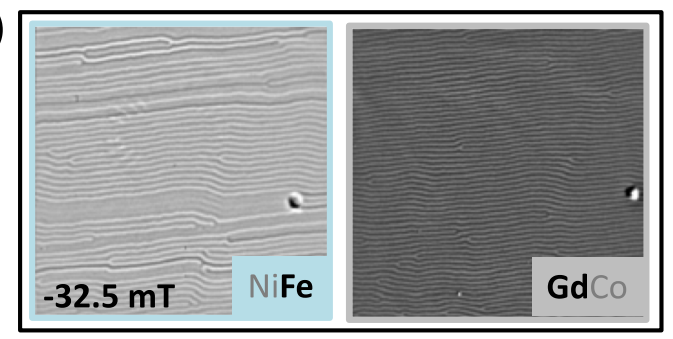

(c)

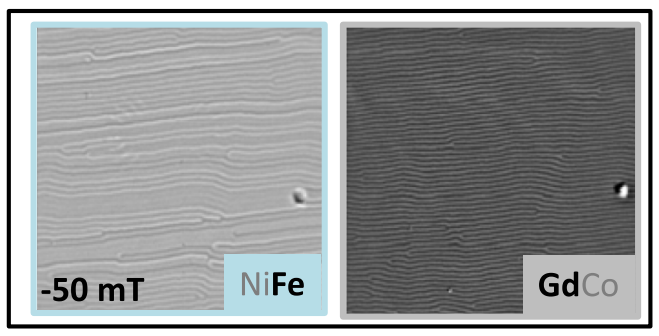

(d)

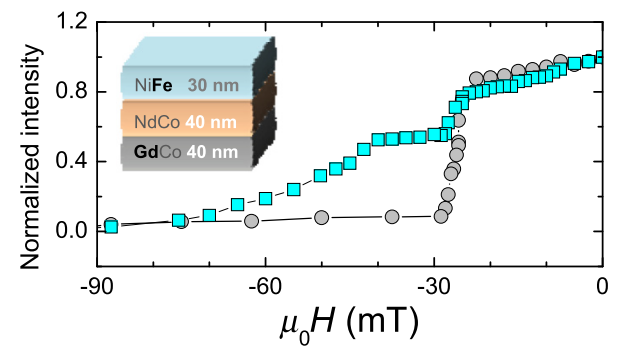

FIG. 4. (a)-(c) $12 \times 12-\mu \mathrm{m}^{2}$ MTXM frames of the magnetization configuration of F30N40G40 along in-plane magnetization reversal measured at the $\mathrm{L}_{3} \mathrm{Fe}$ edge (left panels) and the $\mathrm{M}_{5}$ Gd edge (right panels). Pulsed field amplitude is indicated in each image. In this case, $\mathrm{M}_{5} \mathrm{Gd}$ edge images are acquired along the ascending branch of the hysteresis loop at positive fields of the same amplitude as indicated in each panel. (d) Field dependence of the normalized intensity of the Fe image (squares) and the Gd image (circles) obtained from experimental MTXM images that allow us to reconstruct the element-selective hysteresis loop. 
(a)

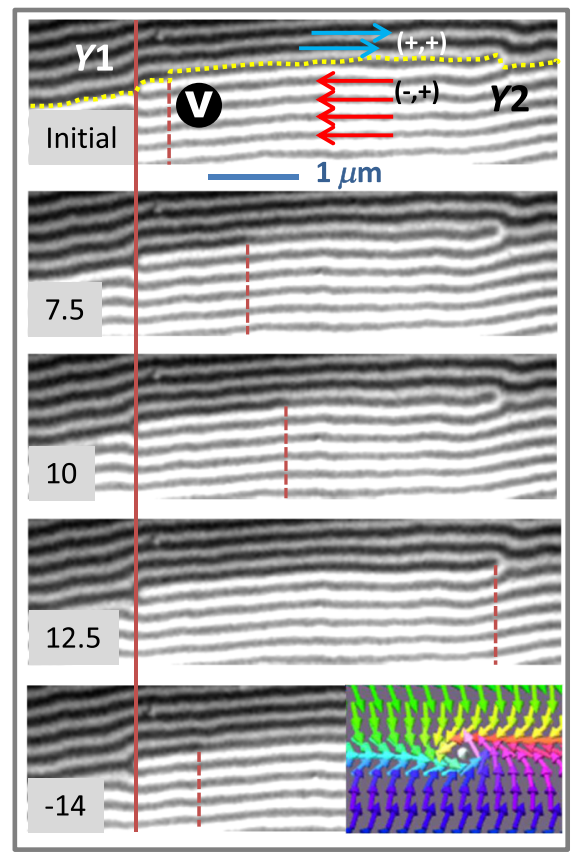

(b)

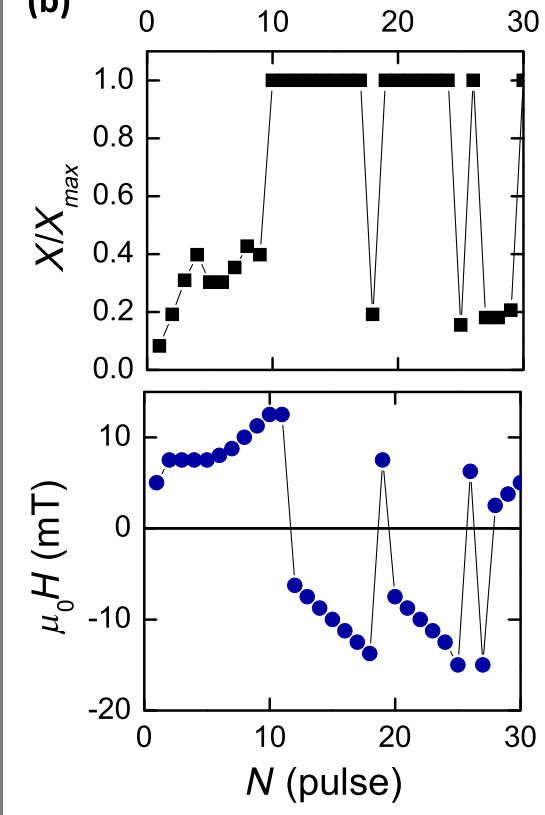

FIG. 5. (a) Detail of MTXM images at the $\mathrm{M}_{5}$ Gd edge of F30N40G40 under a sequence of positive and negative field pulses (field in $\mathrm{mT}$ indicated in the left corner of each panel). Arrows indicate magnetization orientation in black $\left(+M_{x},+M_{z}\right)$ and dark gray $\left(-M_{x},+M_{z}\right)$ stripes. Horizontal dotted line indicates the initial boundary between $+M_{x}$ and $-M_{x}$ domains and vertical dashed lines indicate vortex position (i.e., limit between black $\left(+M_{x},+M_{z}\right)$ and dark gray $\left(-M_{x},+M_{z}\right)$ regions in the central stripe). Inset in the lowest panel is a micromagnetic simulation of a vortex propagating along a $+M_{z}$ stripe at the GdCo bottom surface. (b) Vortex displacement (top panel) and field sequence (bottom panel) showing reversible motion within range. Vortex displacement is in units of $X_{\max }=4$ $\mu \mathrm{m}$, the distance between bifurcations $Y_{1}$ and $Y_{2}$. the vortex jumped back to $X \approx 0.2 X_{\max }$ after a $-14 \mathrm{mT}$ pulse. The remaining pulse sequence showed a reversible motion of the vortex along the stripe from $0.2 X_{\max }$ to $X_{\max }$, depending on pulse amplitude and sign. It is important to mention here that the position of bifurcations $Y_{1}$ and $Y_{2}$ is not altered by the field pulses applied for the present experiment.

In our previous works [11,14], bifurcations within the stripe pattern were identified as nucleation sites for reversed $M_{x}$ domains that expanded away from the bifurcation by the propagation of vortices and antivortices along the stripe-direction. This mechanism gives rise to elongated domains of width $\Lambda$ similar to D1 in Fig. 3(a). Now, the experiment described in Fig. 5 evidences an additional role of bifurcations as pinning sites: as a vortex and/or antivortex reaches a stripe endpoint, further expansion of the reversed domain is hindered. Thus, additional mechanisms must come into play to complete the magnetization reversal of the sample.

\section{Closure and cycloidal domains at coercivity}

As the magnetization reversal process proceeds within the NiFe and GdCo layers, four types of in-plane domains can be identified by comparing experimental MTXM Fe edge images [Fig. 6(a)] with simulated contrast images derived from micromagnetic simulations [Fig. 6(b)] using the formalism described in Ref. [11]. These four domain types are: (i) initial $+M_{x}$ domains characterized by alternating $\left(+M_{x},+M_{z}\right)$ and $\left(+M_{x},-M_{z}\right)$ stripes, i.e., alternating white and dark gray bands; (ii) partially reversed domains with alternating $\left(+M_{x},-M_{z}\right)$ and $\left(-M_{x},+M_{z}\right)$ stripes, i.e., alternating dark gray and light gray bands; (iii) partially reversed domains with alternating $\left(+M_{x},+M_{z}\right)$ and $\left(-M_{x},-M_{z}\right)$ stripes, i.e., alternating white and black bands; (iv) fully reversed $-M_{x}$ domains with alternating $\left(-M_{x},+M_{z}\right)$ and $\left(-M_{x},-M_{z}\right)$ stripes, i.e., alternating light gray and black bands. Figures 6(a)-6(c) display, from top to bottom. these four domain types. The local sense of $M_{y}$ in between stripes is not directly accessible experimentally at the measurement configuration (only sensitive to $M_{x}$ and $M_{z}$ components). However, it can be uniquely determined by the sense of stray-field circulation at the top sample surface (leaving the sample at $+M_{z}$ stripes and entering it at $-M_{z}$ stripes). This allows us to draw the $M_{x}-M_{y}$ sketches for each domain type, shown in Fig. 6(c), which are confirmed by micromagnetic simulations. Thus, partially reversed domains (ii) and (iii) are identified as cycloidal domains with opposite rotation senses.

The fraction of each domain type along the magnetization reversal process is shown in Figs. 6(d) and 6(e) for the NiFe and GdCo layers. At the onset of reversal, the sample is covered by $+M_{x}$ domains, that disappear gradually along the reversal process, with a field dependence very similar to the average intensity in each layer [see solid lines in Figs. 6(d) and 6(e)]. Fully reversed $-M_{x}$ domains follow the opposite trend, appearing at the onset of magnetization reversal and covering the sample at saturation at both the top and bottom layers. However, the prevalence of cycloidal domains is very different at $\mathrm{NiFe}$ and GdCo. In the NiFe case, a significant fraction of cycloidal domains appears (covering up to $30 \%$ of the sample) with a wide stability range from $-22 \mathrm{mT}$ down to $-70 \mathrm{mT}$. At the GdCo layer, the fraction of cycloidal 

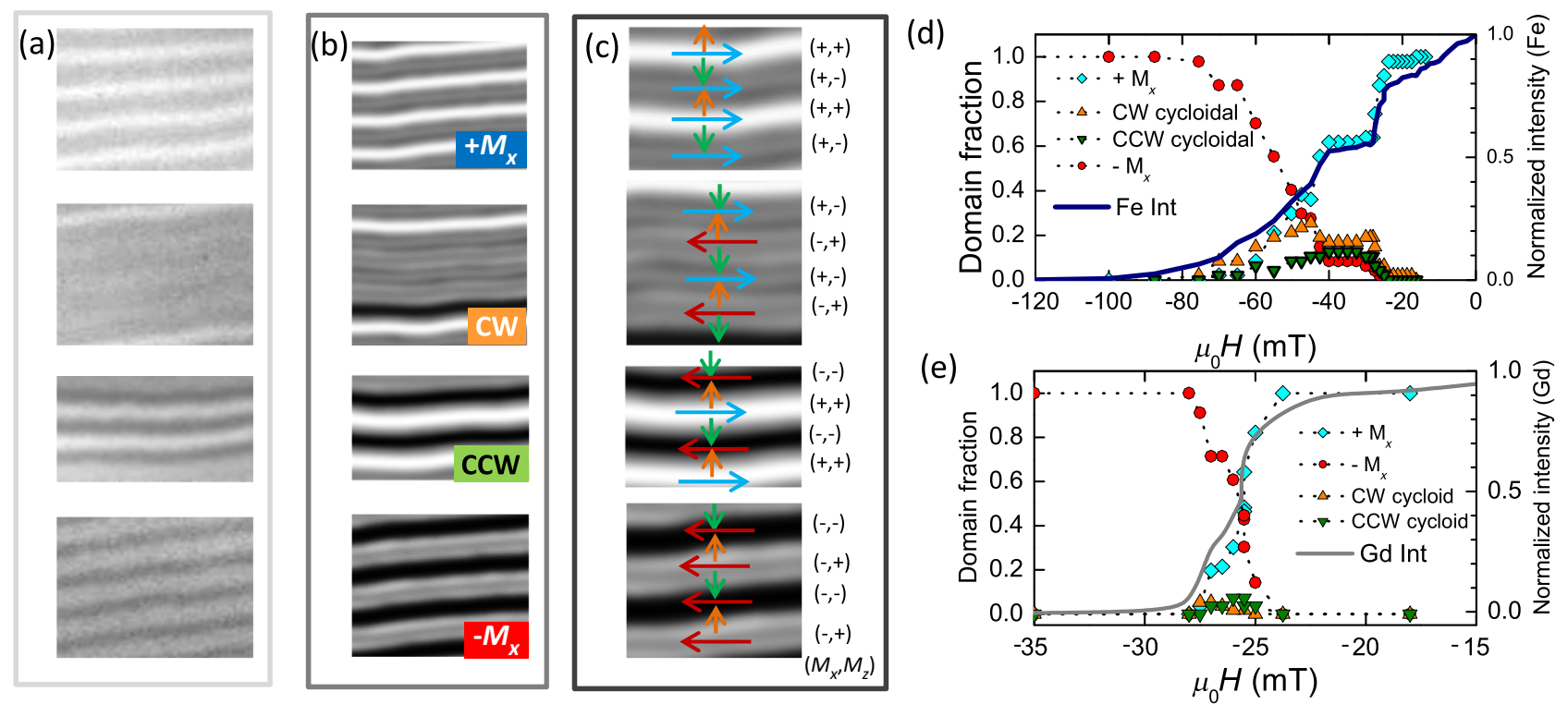

FIG. 6. (a) Four domain types at the NiFe layer (selected from MTXM images of F30N40G40). (b) Simulated MTXM contrast from micromagnetic simulations in different domain configurations. (c) Sketch of in-plane magnetization orientation at each contrast level (making an enlargement of the central regions of panels in (b) for the sake of clarity in the arrow sketches). (d,e) Fraction of each domain type vs reverse field amplitude at (d) the NiFe layer and (e) the GdCo layer. Field dependence of average image contrast at each layer is also shown for comparison (solid line).

domains barely reaches $5 \%$, localized mainly at the boundary between $+M_{x}$ and $-M_{x}$ domains. Thus, the stability of cycloidal domains at the soft NiFe layer appears as the direct cause of the slower reversal process in this layer, making it a suitable material for the controlled propagation of vortex-antivortex pairs in a broad enough field interval.

The stability of cycloidal domains in NiFe and GdCo is related with topological restrictions in the stripe pattern of the central NdCo layer, imprinted by exchange and magnetostatic interactions at the top and bottom layers. Then, it is interesting to note that the best stability range (broadest plateau in the magnetization reversal process) is found for the NiFe layer of F30N40G40 (i.e., the thinnest layer with smallest in-plane anisotropy), as observed both in the macroscopic hysteresis loops (Fig. 2) and in MTXM loops (Figs. 3 and 4). This could be related with the large exchange contribution of cycloidal domains (with $2 \pi$ magnetization rotations on a small length scale), which scales with $\mathrm{NiFe}$ layer thickness favoring their observation in thin magnetic layers. In a similar way, the higher in-plane anisotropy of GdCo would increase the anisotropy contribution in a cycloidal domain configuration, reducing their stability and the effective field range for controlled propagation of vortex-antivortex pairs.

\section{Micromagnetic simulations}

Figure 7(a) shows the field dependence of the magnetization during an in-plane reversal process at each layer obtained from micromagnetic simulations of the F40N60G40 trilayer that qualitatively reproduces the experimental behavior. As the field is reduced from saturation [see inset in Fig. 7(a)] in-plane $M_{x}$ is reduced in each layer due to the development of the stripe-domain pattern with a significant $M_{z}$ oscillation. At remanence, $M_{x}$ is lowest at the central $\mathrm{NdCo}$ layer $\left[\mathrm{M}_{\mathrm{R}}(\mathrm{NdCo})=\right.$ $0.2 M_{S}$ ] but retains relatively large values at the top NiFe $\left[\mathrm{M}_{\mathrm{R}}(\mathrm{NiFe})=0.6 M_{S}\right]$ and bottom $\mathrm{GdCo}\left[\mathrm{M}_{\mathrm{R}}(\mathrm{GdCo})=0.5\right.$ $M_{S}$, where the closure domain structures are localized. For negative fields, reversed domains nucleate at stripe bifurcations and propagate by the correlated motion of vortex-antivortex pairs, as shown in Fig. 7(b1). These initial reversed domains are only one stripe period wide $(\Lambda)$, similar to those observed in Figs. 3(a) and 4(a). This process starts at $-4 \mathrm{mT}$ at $\mathrm{GdCo}$ and $-8 \mathrm{mT}$ at $\mathrm{NdCo}$ and $\mathrm{NiFe}$ and results in a steep decrease of the magnetization down to coercivity $\left(M_{x} \approx 0\right)$.

Depending on bifurcation arrangement in each layer, the vortex-antivortex pair can be dissociated, as shown in Fig. 7(b2) where the antivortex is pinned by interaction with a bifurcation and the vortex has propagated further. In the region between the vortex and the antivortex, the magnetization adopts a cycloidal configuration with alternating $+M_{x}$ and $-M_{x}$ stripes. Individual vortex propagation (uncoupled from its corresponding antivortex) is observed in the experiment of Fig. 5 and is also seen in the NiFe images of Figs. 3(b), 3(c), 4(b), and 4(c), i.e., in the field range where cycloidal domains appear in the sample. 

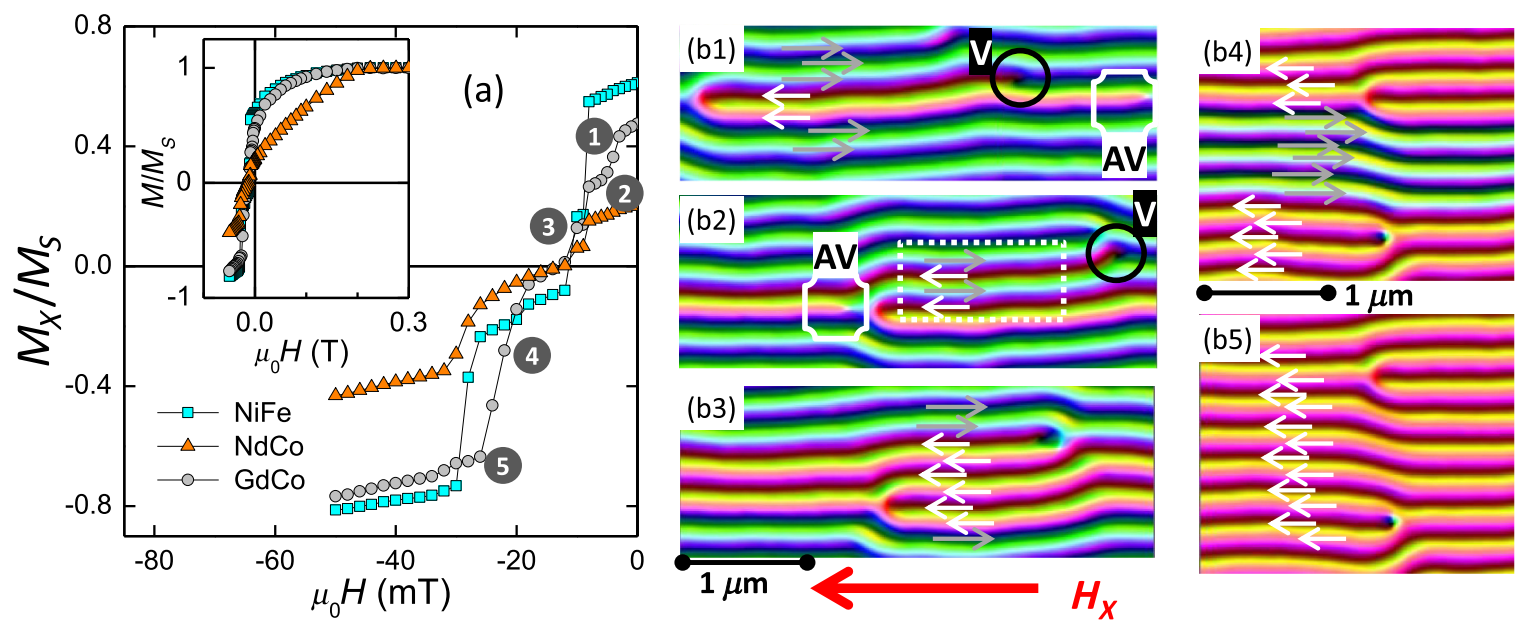

FIG. 7. (a) Element-resolved hysteresis loop, calculated by micromagnetic simulations at F40N60G40. Numbers correspond to images in panel (b). (b) Magnetic configuration of the GdCo layer showing votrex-antivortex pair propagation parallel to stripe direction at the onset of magnetization reversal: (b1) $-4 \mathrm{mT}$, (b2) $-6 \mathrm{mT}$, (b3) $-9 \mathrm{mT}$ [(b2) and (b3) are displaced to the right relative to (b1) to keep track of the propagating vortex-antivortex pair], and domain-wall propagation perpendicular to stripes at the last part of the magnetization reversal: (b4) $-24 \mathrm{mT}$, (b5) $-26 \mathrm{mT}$. Arrows indicate $M_{x}$ sense in each stripe. Note the cycloidal domain in (b2) due to pinning of an antivortex at the bifurcation, highlighted by the dotted rectangle. Also, a certain displacement of bifurcations along the stripe directions can be observed between (b2) and (b3).

As the negative field increases, vortices and antivortices propagate guided by the stripe direction until they are stopped by stripe endpoints [as shown in Fig. 7(b3)]. At the simulated sample, the number of bifurcations is relatively small (between four and six), so that a stable magnetization configuration with all vortices pinned at stripe endpoints is easily reached both for the NiFe and GdCo layers. It results in a magnetization plateau just below coercivity that extends over a $\Delta \mu_{0} H=6 \mathrm{mT}$ interval at GdCo and over $\Delta \mu_{0} H=14 \mathrm{mT}$ at the NiFe layer.

Finally, a steep decrease of $M_{x}$ is observed starting at $-20 \mathrm{mT}$ for GdCo and at $-28 \mathrm{mT}$ at the NiFe layer. It corresponds to the activation of an additional magnetizationreversal mechanism by the propagation of a domain wall in the $x-z$ plane along the $y$ direction, i.e., perpendicular to the direction of the stripes [see Figs. 7(b4) and 7(b5)], indicating that vortex-antivortex propagation has ceased to be confined along the stripes. At this point of the hysteresis loop, the sample surface is fully covered by $-M_{x}$ domains. The remaining part of the reversal process corresponds to the approach to $-M_{x}$ saturation as the amplitude of the $M_{z}$ oscillation in the stripe pattern is reduced for large negative $H_{x}$ fields.

Spin rotations in the simulated closure domain patterns can be seen in more detail in Fig. 8. $+M_{x}$ closure domains [circles in Fig. 8(a) and the sketch in Fig. 8(b)] combine an in-plane $\left(M_{x}-M_{y}\right) \pi$ rotation correlated with a $M_{z}$ oscillation of reduced amplitude $\pm 0.5 M_{S}$, so that the magnetization is always constrained to the $+M_{x}$ hemisphere. The configuration of $-M_{x}$ closure domains is equivalent but with magnetization in the $-M_{x}$ hemisphere [squares in Fig. 8(a) and the sketch in Fig. 8(d)]. Partially (a)

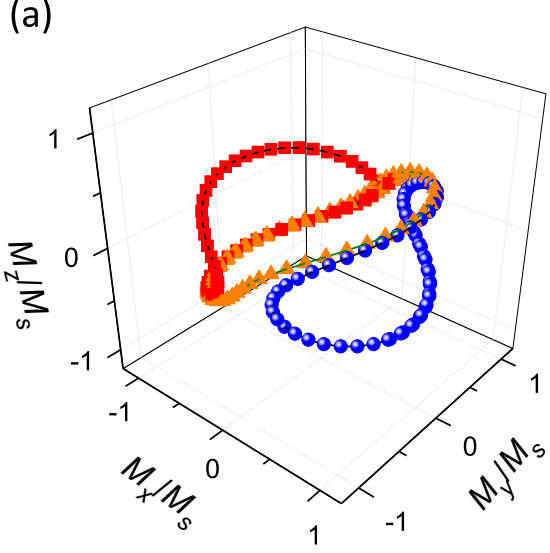

(b) $+M_{x}$ (c) Cycloidal

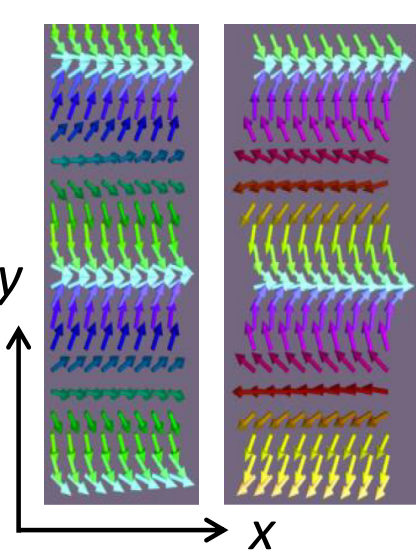

(d) $-M_{x}$

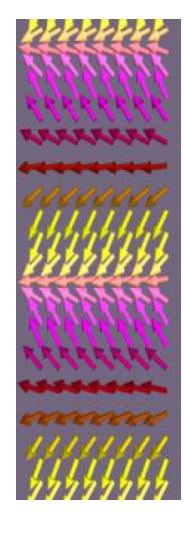

FIG. 8. (a) Spin rotation at the bottom GdCo, derived from the micromagnetic simulations in Fig. 7: blue circles, $+M_{x}$ domain; orange triangles, cycloidal; red squares, $-M_{x}$ domain. The corresponding magnetic configurations are shown in (b), (c), and (d). 
reversed domains are a combination of an in-plane cycloid ( $2 \pi$ rotation in the $M_{x}-M_{y}$ plane rotation) with a correlated $M_{z}$ oscillation [triangles in Fig. 8(a) and the sketch in Fig. 8(c)]. Two possible rotation senses can appear depending on the relative phase between the in-plane and out-of-plane spin rotations, as observed experimentally.

The comparison between micromagnetic simulations and the experimental MTXM images shows that the observation of partially reversed cycloidal domains requires two basic ingredients: (1) Effective vortex and antivortex guiding along the stripe-domain pattern, i.e., that the propagation of domain walls transverse to the stripes is delayed to high enough magnetic fields. (2) The dissociation of vortex-antivortex pairs as they propagate along the stripe pattern. This can occur by interaction with stripe bifurcations [as seen in Fig. 7(b2)] or other defects present in the sample (as seen in the experimental images in Figs. 3 and 4). Experimentally, these conditions are clearly met only at the NiFe layer but not at the GdCo layer.

Thus, the observation of cycloidal domains in the experimental MTXM images can be used to characterize the field range where vortices and antivortices are effectively guided by the stripe pattern, which is of the order of tens of $\mathrm{mT}$ for the NiFe layer. This is an interesting result since the existence of a broad enough field interval of effective vortex guiding is essential for possible applications of the deterministic propagation of vortex-antivortex pairs found in Ref. [11].

\section{CONCLUSIONS}

In summary, cycloidal domains are identified in NiFe layers during the magnetization reversal process of $\mathrm{NiFe} / \mathrm{NdCo} / \mathrm{GdCo}$ trilayers by the comparison of MTXM images and micromagnetic simulations. The stripe pattern of the central WPMA NdCo layer imprints a periodic closure domain structure at the top and bottom NiFe and GdCo layers where magnetization reversal is initiated at stripe bifurcations, by the nucleation of vortex-antivortex pairs. It is found that, in the NiFe layer, there is a field range around coercivity (of several tens of $\mathrm{mT}$ ) in which pairs of vortices and antivortices propagate preferentially along the stripes and, then, they dissociate while pinning at stripe endpoints and sample imperfections. The sensitivity of MTXM images to $M_{z}$ and $M_{x}$ components at the different layers reveals that, at that point of the hysteresis loop, cycloidal domains with alternating $M_{x}$ sign appear in the sample, covering up to $30 \%$ of the NiFe surface. The stability range of cycloidal domains gives a measure of the field interval in which propagation of vortices and antivortices within the NiFe layer can be effectively controlled by the stripe pattern.

\section{ACKNOWLEDGMENTS}

Work supported by Spanish MINECO [Grants No. FIS 2013-45469 and No. FIS2016-76058 (AEI/FEDER,EU)] and by FICYT-Asturias (Grant No. FC-GRUPIN14-040). We thank J. Avila (Alba staff) for assistance in the pulsed magnetic field set up. We thank Unidad de Medidas magnéticas y RMN de Sólidos de los Servicios Cientifico Técnicos of Universidad de Oviedo for the VSM characterization. A.H.-R. acknowledges the support from European Union's Horizon 2020 research and innovation programme under Marie Skłodowska-Curie Grant No. H2020-MSCAIF-2016-746958.

[1] U. K. Rößler, A. N. Bogdanov, and C. Pfleiderer, Spontaneous skyrmion ground states in magnetic metals, Nature 442, 797 (2006).

[2] X. Yu, M. Mostovoy, Y. Tokunaga, W. Zhang, K. Kimoto, Y. Matsui, Y. Kaneko, N. Nagaosa, and Y. Tokura, Magnetic stripes and skyrmions with helicity reversals, Proc. Natl Acad. Sci. USA 109, 8856 (2012).

[3] F. Zheng, H. Li, S. Wang, D. Song, C. Jin, W. Wei, A. Kovács, J. Zang, M. Tian, Y. Zhang, H. Du, and R. E. Dunin-Borkowski, Direct Imaging of a Zero-Field Target Skyrmion and Its Polarity Switch in a Chiral Magnetic Nanodisk, Phys. Rev. Lett. 119, 197205 (2017).

[4] F. N. Rybakov, A. B. Borisov, S. Blügel, and N. S. Kiselev, New spiral state and skyrmion lattice in 3D model of chiral magnets, New J. Phys. 18, 045002 (2016).

[5] L. Schmidt, J. Hagemeister, P.-J. Hsu, A. Kubetzka, K. von Bergmann, and R. Wiesendanger, Symmetry breaking in spin spirals and skyrmions by in-plane and canted magnetic fields, New J. Phys. 18, 075007 (2016).

[6] S. A. Montoya, S. Couture, J. J. Chess, J. C. T. Lee, N. Kent, D. Henze, S. K. Sinha, M.-Y. Im, S. D. Kevan, P. Fischer, B. J. McMorran, V. Lomakin, S. Roy, and E. E. Fullerton, Tailoring magnetic energies to form dipole skyrmions and skyrmion lattices, Phys. Rev. B 95, 024415 (2017).

[7] C. Blanco-Roldán, C. Quirós, A. Sorrentino, A. HierroRodríguez, L. M. Álvarez Prado, R. Valcárcel, M. Duch, N. Torras, J. Esteve, J. I. Martín, M. Vélez, J. M. Alameda, E. Pereiro, and S. Ferrer, Nanoscale imaging of buried topological defects with quantitative X-ray magnetic microscopy, Nat. Commun. 6, 8196 (2015).

[8] Y. Zhou, E. Iacocca, A. A. Awad, R. K. Dumas, F. C. Zhang, H. B. Braun, and J. Åkerman, Dynamically stabilized magnetic skyrmions, Nat. Commun. 6, 8193 (2015).

[9] J. Li, K. W. Moon, A. Doran, M. A. Marcus, A. T. Young, E. Arenholz, S. Ma, R. F. Yang, C. Hwang, and Z. Q. Qiu, Tailoring the topology of an artificial magnetic skyrmion, Nat. Commun. 5, 4704 (2014).

[10] S. Wintz, C. Bunce, A. Neudert, M. Körner, T. Strache, M. Buhl, A. Erbe, S. Gemming, J. Raabe, C. Quitmann, and J. Fassbender, Topology and Origin of Effective Spin Meron Pairs in Ferromagnetic Multilayer Elements, Phys. Rev. Lett. 110, 177201 (2013). 
[11] A. Hierro-Rodriguez, C. Quirós, A. Sorrentino, R. Valcárcel, I. Estébanez, L. M. Alvarez-Prado, J. I. Martín, J. M. Alameda, E. Pereiro, M. Vélez, and S. Ferrer, Deterministic propagation of vortex-antivortex pairs in magnetic trilayers, Appl. Phys. Lett. 110, 262402 (2017).

[12] S. Fust, S. Mukherjee, N. Paul, J. Stahn, W. Kreuzpaintner, P. Böni, and A. Paul, Realizing topological stability of magnetic helices in exchange-coupled multilayers for all-spin-based system, Sci. Rep. 6, 33986 (2016).

[13] A. Hubert and R. Schäfer. Magnetic Domains: The Analysis of Magnetic Microstructures (Springer-Verlag, Berlin, 1998).

[14] A. Hierro-Rodriguez, C. Quirós, A. Sorrentino, C. BlancoRoldán, L. M. Alvarez-Prado, J. I. Martín, J. M. Alameda, E. Pereiro, M. Vélez, and S. Ferrer, Observation of asymmetric distributions of magnetic singularities across magnetic multilayers, Phys. Rev. B 95, 014430 (2017).

[15] M. Manfrini, J.-V. Kim, S. Petit-Watelot, W. Van Roy, L. Lagae, C. Chappert, and T. Devolder, Propagation of magnetic vortices using nanocontacts as tunable attractors, Nat. Nanotechnol. 9, 121 (2014).

[16] S. Zhang, A. A. Baker, S. Komineas and T. Hesjedal, Topological computation based on direct magnetic logic communication, Sci. Rep. 5, 15773 (2015).

[17] L. D. Geng and Y. M. Yin, Magnetic vortex racetrack memory, J. Magn. Magn. Mater. 423, 84 (2017).

[18] F. Li, T. Nattermann, and V. L. Pokrovsky, Vortex Domain Walls in Helical Magnets, Phys. Rev. Lett. 108, 107203 (2012).

[19] S. Meckler, N. Mikuszeit, A. Preßler, E.Y. Vedmedenko, O. Pietzsch, and R. Wiesendanger, Real-Space Observation of a Right-Rotating Inhomogeneous Cycloidal Spin Spiral by Spin-Polarized Scanning Tunneling Microscopy in a Triple Axes Vector Magnet, Phys. Rev. Lett. 103, 157201 (2009).

[20] M. Haze, Y. Yoshida, and Y. Hasegawa, Experimental verification of the rotational type of chiral spin spiral structures by spin-polarized scanning tunneling microscopy, Sci. Rep. 7, 13269 (2017).

[21] I. Gross, W. Akhtar, V. Garcia, L. J. Martínez, S. Chouaieb, K. Garcia, C. Carretero, A. Barthélémy, P. Appel, P. Maletinsky, J.-V. Kim, J. Y. Chauleau, N. Jaouen, M. Viret, M. Bibes, S. Fusil, and V. Jacques, Real-space imaging of non-collinear antiferromagnetic order with a single-spin magnetometer, Nature 549, 252 (2017).

[22] N. Mikuszeit, S. Meckler, R. Wiesendanger, and R. Miranda, Magnetostatics and the rotational sense of cycloidal spin spirals, Phys. Rev. B 84, 054404 (2011).

[23] A. Hierro-Rodriguez, R. Cid, M. Vélez, G. RodriguezRodriguez, J. I. Martín, L. M. Alvarez- Prado, and J. M. Alameda, Topological Defects and Misfit Strain in Magnetic Stripe Domains of Lateral Multilayers With Perpendicular Magnetic Anisotropy, Phys. Rev. Lett. 109, 117202 (2012).

[24] C. Blanco-Roldán, Y. Choi, C. Quirós, S. M. Valvidares, R. Zarate, M. Vélez, J. M. Alameda, D. Haskel, and J. I. Martín, Tuning interfacial domain walls in $\mathrm{GdCo} / \mathrm{Gd} /$ GdCo' spring magnets, Phys. Rev. B 92, 224433 (2015).

[25] E. Pereiro, J. Nicolas, S. Ferrer, and M. R. Howells, A soft X-ray beamline for transmission X-ray microscopy at ALBA, J. Synchrotron Radiat. 16, 505 (2009).

[26] A. Sorrentino, J. Nicolás, R. Valcárcel, F. J. Chichón, M. Rosanes, J. Avila, A. Tkachuk, J. Irwin, S. Ferrer, and E. Pereiro, MISTRAL: A transmission soft X-ray microscopy beamline for cryo nano-tomography of biological samples and magnetic domains imaging, J. Synchrotron Radiat. 22, 1112 (2015).

[27] A. Vansteenkiste, J. Leliaert, M. Dvornik, M. Helsen, F. Garcia-Sanchez, and B. Van Waeyenberge, The design and verification of MuMax3, AIP Adv. 4, 107133 (2014).

[28] G. E. Rowland, Muview, http://www.grahamerowlands.com/ main/muview. 